\title{
Posibilidades y limitaciones de la educación intercultural en contexto mapuche ${ }^{1}$
}

\section{Possibilities and limitations of the intercultural education in mapuche context}

Aceptación: Diciembre - 2008

Dr. Segundo Quintriqueo M.*

Aprobación: Marzo - 2009

\section{RESUMEN}

El presente artículo tiene por objeto la educación intercultural en contexto mapuche, donde se exponen antecedentes teóricos y prácticos referidos a su origen en América Latina y Chile, asociado al carácter histórico, monocultural y centralizado de la educación. La educación intercultural es definida en relación a las demandas históricas de las familias y comunidades para mejorar la educación y valorar el patrimonio cultural mapuche en la educación escolar. Como resultado se presenta un análisis sobre las posibilidades y limitaciones de la educación intercultural en relación a la coexistencia de racionalidades: mapuche y no mapuche en contexto intercultural. Estas racionalidades generan una distancia epistemológica en la definición de contenidos y finalidades educativas para la formación de persona en contexto de relaciones interétnicas e interculturales.

PALABRAS CLAVE: Educación intercultural, contexto mapuche, currículum intercultural

\section{ABSTRACT}

This article has as a main objective intercultural education in mapuche context, where theoretical and practical antecedents are exposed, referring to their origin in Latin America and Chile, which is associated to the historical, monocultural and centered character of education. Intercultural education is defined in relation to the historical demands of families and communities in order to improve education and value cultural mapuche heritage on school education. As a result it is presented an analysis about the possibilities and limitations of intercultural education in relation to the coexistence of rationalities: mapuche and non mapuche in intercultural context. These rationalities generate an epistemological distance on the definition of the educational contents and objectives for the formation of the person in the context of interethnic and intercultural relationships.

KEY WORDS: intercultural education, mapuche context, intercultural curriculum

\footnotetext{
* Académico Escuela de Educación Básica, Facultad de Educación, Universidad Católica de Temuco. Correo: squintri@uct.cl

1 El artículo comunica un aspecto de la Tesis Doctoral “Implicancias de un modelo curricular monocultural en la construcción de la identidad sociocultural de alumnos/as mapuches de la IX región de La Araucanía” (Quintriqueo, 2007) y al proyecto Fondecyt $\mathrm{n}^{\circ} 1085293$ "Racionalidad del método educativo mapuche, desde la memoria social de kimches: fundamentos para una educación intercultural".
} 


\section{INTRODUCCIÓN}

La investigación sostiene como hipótesis que los kimches -personas sabias- y padres de familias conocen los fundamentos, contenidos y finalidades educativas para la formación de persona en saberes y conocimientos mapuches ${ }^{1}$. Sin embargo, históricamente, la escolarización de niños mapuches se realiza sobre la base de contenidos y finalidades educativas monoculturales occidentales. Cuando los niños se incorporan a la educación escolar, adquieren un conjunto de contenidos y finalidades educativas, objetivos de enseñanza y aprendizaje, que se fundamentan en lógicas de saberes y conocimientos educativos no mapuches. Al no explicitarse ambas lógicas de conocimientos, occidental y mapuche, se genera en los alumnos problemas en el aprendizaje de contenidos, en el cumplimiento del trabajo escolar y la comunicación intercultural. El conocimiento occidental está relacionado con ciertas categorías del saber disciplinar consignado en los sectores y subsectores de aprendizaje del currículum escolar. El conocimiento mapuche está relacionado con un conjunto de saberes representados en la memoria individual y social de la familia y la comunidad. Ambas categorías y racionalidades de conocimientos mapuche y occidental son objeto de estudio para la generación de una base de conocimientos que fundamenten la educación intercultural.

La educación intercultural en América Latina tiene sus orígenes en países como Guatemala, Ecuador, Perú, Colombia, México y Bolivia (Chiodi, 1990; Moya, 1996). En dicho contexto, y también en Chile, la educación intercultural se relaciona con las demandas de los Pueblos Indígenas para tener mejores oportunidades y el acceso a una mejor calidad de la educación. Entonces, se fundamenta en el marco de las relaciones interétnicas e interculturales, marcada por el contacto histórico entre mapuches y no mapuches, donde las decisiones sobre políticas educacionales tienen un carácter centralizado, desde organismos centrales creados por el Estado. Primero, por las organizaciones coloniales, y segundo, en el marco de la configuración del Estado. El sistema educacional desde estos dos momentos de la historia, ha privilegiado sistemas culturales dominantes, donde no se consideran las culturas originarias. Este es un proceso similar en los sistemas educacionales en Chile y América Latina. Así, en el marco de la formación escolar y las relaciones sociales, se ha creado una cultura oficial y otras marginales o periféricas (Magendzo, 1986). El carácter histórico monocultural y centralizado de la educación en Chile, particularmente en el subsistema de Educación Básica, se mantiene hasta hoy día, aun cuando en las últimas cinco décadas ha surgido una consideración de la diversidad étnica y cultural en el campo de la educación en el país (Catriquir y Durán, 1997; Magendzo, 2007).

El carácter monocultural de la educación deviene desde de la constitución del Estado-nación chileno en el siglo XIX, donde se enfatizan los principios culturales de civilización, centralismo político y unidad nacional (Egaña, 2000; Magendzo, 2007), expresados en el reconocimiento de una cultura única oficial y legitimada social e institucionalmente por la escuela (Rokwell, 1995; Perrenoud, 2005; Quintriqueo, 2007). Este modelo educativo se ha configurado teniendo como referente el primer mundo (Labarca, 1939), en cuyo proceso no se reconocen las culturas originarias al interior de la sociedad nacional.

Solo a partir de los noventa se aprecia una orientación hacia la consideración de las diferencias culturales y étnicas en el subsistema de Educación Básica, aunque con políticas específicas, sin modificar el sistema en su conjunto. Estas políticas se operacionalizan a través del currículum, que expresa los mismos rasgos antes expuestos: monocultural, centralizado y homogeneizante, lo cual se observa en diversos instrumentos oficiales tales como: 1) La Ley de Disposición Fundamental sobre la materia, de 1813, que reglamenta la Educación Primaria; 2) La Ley de Instrucción Primaria, de 1860; 3) La Ley de Enseñanza

Un avance del artículo ha sido presentado en el Ciclo de Conferencia REUNA en el marco de la proyección al medio del CENTRO DE INVESTIGACIÓN EN EDUCACIÓN EN CONTEXTO INDÍGENA E INTERCULTURAL (CIECII), de Iniciativa Científica Milenio (ICM). Universidad Católica de Temuco. 
Obligatoria, de 1920; 4) La Ley Orgánica Constitucional, de 1990; y 5) Programas educacionales de las diferentes reformas (Labarca, 1939; Egaña, 2000). En ese contexto, las políticas educacionales han sido estudiadas en Chile por Brunner (1988) y Magendzo (1986); en América Latina por Tedesco (2000), y en otras partes del mundo por Forquin (1997) y Perrenoud (2005), donde se exponen las desigualdades sociales, culturales, educacionales y económicas como fenómenos asociados a políticas educativas y curriculares que no consideran las diferencias. En su defecto, estas políticas educacionales generan una distancia cada vez mayor no solo entre los grupos socioeconómicos más bajos y aquellos más altos en Chile y América Latina, sino también en la distancia epistemológica y política en las diferencias socioculturales y étnicas (Tedesco, 2000; Quintriqueo, 2007).

El currículum escolar chileno es un reflejo del modelo cultural occidental, particularmente el educativo, que ha escolarizado a muchas generaciones de mapuches desde la masificación de la escuela, respondiendo al imaginario nacional que ha involucrado a diversos sectores de la sociedad, generando una relación hegemónica del saber occidental con relación al saber mapuche. En este sentido, los documentos oficiales y recursos educativos no solo movilizan estereotipos, sino también estados de silenciamiento y ocultamiento. Producto de este proceso, las generaciones jóvenes de mapuches y no mapuches manifiestan su desconocimiento respecto de la sociedad y cultura mapuche. Un impacto de estos fenómenos de negación y ocultación, son los grados en que los individuos asumen o rechazan su identidad étnica, como uno de los aspectos centrales en la transformación de la familia y la comunidad (Quilaqueo y Quintriqueo, 2008).

En ese contexto, las demandas educacionales de los pueblos originarios y propuestas del Estado chileno se relacionan con la necesidad de una educación diferenciada (Catriquir y Durán, 1997). Esta demanda progresivamente se ha ido perfeccionando, hasta plantear la necesidad de incluir la diversidad sociocultural y étnica en los diversos niveles del sistema educacional: Pre-escolar, Básico, Medio y Superior. En este proceso, la demanda por una educación diferenciada, no solo busca superar la educación uniforme, etnocéntrica y nacionalista, sino que además, la revisión de programas de renovación curricular que focalice la incorporación del medio, la cultura y principalmente a la persona (Catriquir y Durán, 1997). Por su parte, las demandas que las organizaciones indígenas plantean, desde los inicios del siglo XX hasta la década de los setenta, se centra en la incorporación de la lengua mapuche mapunzugun en el sistema escolar (Foerster, 1988; Canales, 1998). Hacia fines de los 80, la demanda se refiere a una Educación Intercultural Bilingüe (Documento, acuerdo de Nueva Imperial, 1989). El propósito es superar procesos de homogeneización nacional y la asimilación forzada en el ámbito político, cultural, social y educacional (MIDEPLAN, 2004; UNESCO, 2005). Desde la década de los ochenta en adelante se instala el "debate público por la autonomía, el reconocimiento constitucional y el derecho a la libre determinación" (MIDEPLAN, 2004:94). Los instrumentos jurídicos internacionales que vienen a sustentar estas demandas políticas son el Convenio 169 de la OIT y la Declaración de la Naciones Unidas sobre los derechos de los pueblos indígenas (INSTITUTO INDÍGENA INTERAMERICANO, 1996), la Ley Indígena $N^{\circ} 19.253$ y la Ley Orgánica Constitucional de Enseñanza (LOCE, 1990) № 18.944, constituyen las primeras bases para la Educación Intercultural Bilingüe (EIB). En el ámbito educativo, esta última ley, en su artículo $\mathrm{N}^{\circ} 28$, señala que es preciso incorporar en los programas de estudio nacional una unidad que permita a los alumnos conocer adecuadamente las culturas indígenas, sus idiomas y la valoración de Estas. A ellos se agrega el uso y conservación de los idiomas indígenas junto al español en las áreas de alta densidad indígena. En este sentido, plantea establecimiento de unidades programáticas que posibiliten a los educandos el aprendizaje de su propia cultura e idioma, para valorarse positivamente tanto en el medio escolar, comunitario y la sociedad global. También, se consigna el compromiso que deben tener las instituciones universitarias en promocionar y establecer cátedras de historia, cultura y lenguas indígenas en el currículum de la formación universitaria.

Entonces, la EIB se concibe como un enfoque educativo que permite enriquecer la 
formación de persona y la calidad de la educación para todos los actores involucrados: indígenas y no indígenas (Cañulef, 1998; Chiodi y Bahamondes, 2002; Quintriqueo, 2007). Por su parte, la educación intercultural tiene como centro la relación y comunicación necesaria entre personas y grupos sociales que tienen representaciones diferentes sobre fenómenos socioculturales marcados por el contacto interétnico e intercultural y reproducido a través de procesos de socialización. Uno de los problemas que aborda la educación intercultural es la incorporación de la lengua indígena al sistema escolar y con ella busca la pertinencia en la enseñanza del castellano como segunda lengua; de igual modo, se orienta hacia el desarrollo de la lengua indígena en el sistema escolar. En el ámbito de la política pública, la educación intercultural se incorpora en el marco del proceso de innovación. Por ejemplo, el Ministerio de Educación chileno plantea la educación intercultural como una política para asegurar igualdad de oportunidades, mejorar la calidad de los aprendizajes de los integrantes de las etnias reconocidas por el Estado, ampliando la oferta educativa desde una concepción pluricultural, a fin de contribuir y favorecer las prácticas que fortalezcan las costumbres y formas de entender el mundo de un significativo sector de nuestra sociedad (MINEDUC, 2005).

\section{METODOLOGÍA}

La investigación es de carácter descriptiva (Rodríguez, Gil y García, 1999) y se inscribe en el marco de la Metodología de la Investigación Educativa (Gauthier et al., 1998; Bisquerra, 2004). Los enfoques metodológicos retenidos son: 1) La investigación socioeducacional (Bonal, 1998; Gauthier, 1998; Fernández, 2003); y 2) El análisis sociohistórico (Durán y Cárdenas, 1983; Egaña, 2000). La investigación se realiza en el contexto de seis escuelas de Educación Básica de la IX Región, situadas en comunidades mapuches. Las escuelas son: Tres Cerros, Ñielol y Truf-Truf de la comuna de Padre Las Casas; El Crucero de la comuna de Temuco; Huentelar y San Lorenzo de la comuna de Nueva Imperial.

En el medio escolar, la técnica de muestreo empleada es intencional, por conglome- rado no probabilístico (Bisquerra, 2004). En el conglomerado alumnos(as) se considera una muestra total de 268 sujetos. De estos, 109 corresponden a la escuela Ñielol, 50 a la escuela Tres Cerros, 32 a la escuela Truf-Truf, 14 a la escuela Huentelar, 24 a la escuela San Lorenzo y 39 a la escuela El Crucero. Considera alumnos de $5^{\circ}$ a $8^{\circ}$ año de Educación Básica, debido a que tienen niveles básicos de lectura, para responder en forma adecuada el cuestionario que se considera en la investigación. En las escuelas que conforman la muestra, el $92,5 \%$ de la población escolar corresponde a alumnos de ascendencia mapuche. En el conglomerado padres se considera una muestra de 167 sujetos.

Para la recolección de la información, en el medio escolar se aplica un cuestionario con escala de valoración Likert a 268 alumnos, 167 padres y 21 profesores, en las seis escuelas que considera la investigación. En el medio comunitario se aplican entrevistas individuales a 16 kimches, con preguntas semiestructuradas y semidirigidas (Quivy y Campenhoudt, 1998).

El procesamiento y análisis de los datos se realiza mediante resúmenes de casos y una descripción cualitativa de las frecuencias, a través del sistema de análisis SPSS. Los datos son tratados en base a una codificación abierta y axial (Bisquerra, 2004), en relación a las categorías y dimensiones que están en la base del modelo de análisis. El análisis de los datos es descriptivo a través del Análisis de Contenidos (AC) que busca relevar núcleos de saberes centrales de orden abstracto, dotados de sentido y significado desde la perspectiva de los sujetos que se consideran de la muestra, a partir de contenidos concretos expresados en el texto. Este método opera en dos fases: primero, la producción de significados mediante construcción de un sistema jerárquico de categorías que deriva de la combinación del Método de Comparaciones Constantes (MCC) y el Análisis por Unidades Temáticas (AUT) (Kippendorff, 1990; Quivy y Campenhoudt, 1998). En este proceso se construye una teoría fundamentada en relación a los significados culturales de los datos con respecto a los contenidos centrales y relevantes (Kippendorff, 1990). 


\section{PRESENTACIÓN DE RESULTADOS}

Las posibilidades y limitaciones de la educación intercultural se relacionan con: 1) La valoración del saber y conocimiento mapuche como elemento de identidad sociocultural; 2) La valoración del mapunzugun como herramienta de dominio y comunicación del conocimiento mapuche; y 3) La presencia de saberes y conocimientos mapuches para mejorar procesos de aprendizaje en el medio escolar.

En el marco de la educación intercultural, la identidad sociocultural de los estudiantes, asociada a los elementos de la cultura y la comunidad mapuche, están en la base de la identidad individual y se expresa en el nombre de la familia, el conocimiento sobre el mapunzugun y los parientes de la comunidad. El saber y conocimiento que se enseña en la escuela están consignados en el marco curricular y aquellos que los profesores portan como producto de la socialización en el marco de la cultura occidental. Al interrogar si "El saber que se enseña en la escuela, tiene más valor que el saber mapuche", se constata que el $52 \%$ de los alumnos y el $53 \%$ de los padres atribuyen una valoración de acuerdo o muy de acuerdo. Esto significa que los alumnos como los padres tienen conciencia que eso que se aprende en la escuela tiene mayor valor que los saberes sociales que existen en la familia y la comunidad. En cambio, los profesores sostienen que el saber que se enseña en la escuela no tiene más valor que el saber mapuche, atribuyendo un $62 \%$ de valoración en desacuerdo o muy en desacuerdo. Esto significa que los profesores son conscientes que en el medio escolar coexisten varios tipos de saberes, o bien, que existe un espacio para la enseñanza del saber mapuche.
En el mismo sentido, al preguntar sobre la presencia de conocimiento mapuche en la escuela, el $72 \%$ de los alumnos y el $67 \%$ de los profesores atribuyen una valoración de acuerdo o muy de acuerdo en que "La enseñanza escolar permite conocer y apreciar a la gente del pueblo mapuche". Significa que la escuela utiliza distintos mecanismos para que los alumnos, sean mapuche o no, logren un conocimiento y cierto aprecio sobre la gente mapuche en el contexto regional. Sin embargo, el $72 \%$ de los padres atribuyen una valoración en desacuerdo o muy en desacuerdo en que la identidad mapuche se aprende en la escuela. Esto significa que los padres son conscientes que la identidad mapuche los niños la aprenden fundamentalmente en el medio familiar y comunitario.

Sorprendentemente, al interrogar sobre la identidad de los niños en el medio escolar, el $73 \%$ de los alumnos y el $76 \%$ de los padres atribuyen una valoración de acuerdo o muy de acuerdo que "En la escuela me identifico como mapuche por mi(s) apellido(s)". Significa que para los alumnos como para los padres, en la escuela los niños en forma explícita o implícita reciben un conocimiento sobre los mapuches, lo cual les permite identificarse como perteneciente a la familia y la comunidad. Es decir, el apellido como un conocimiento parental es un elemento fundamental para la identificación de los niños y adolescentes. Por su parte, el $100 \%$ de los profesores atribuyen una valoración en desacuerdo o muy en desacuerdo con respecto al mismo aspecto. Esto significaría, según la apreciación del profesorado, el conocimiento sobre los parientes asociado al uso del mapunzugun no constituye un elemento de identificación mapuche para los niños y adolescentes en la escuela (Véase Tabla $\mathrm{N}^{\circ}$ 1). 
Tabla $N^{\circ}$ 1. La valoración del saber y conocimiento mapuche en el medio escolar

\begin{tabular}{|c|c|c|c|c|c|}
\hline \multirow[b]{2}{*}{ Categorías } & \multirow[b]{2}{*}{ Actores } & \multicolumn{3}{|c|}{ Escala de valoración } & \multirow[b]{2}{*}{$\%$} \\
\hline & & No sé & $\begin{array}{c}\text { En desacuerdo } \\
\text { o } \\
\text { Muy en desacuerdo }\end{array}$ & $\begin{array}{c}\text { De acuerdo } \\
\text { o } \\
\text { Muy de acuerdo }\end{array}$ & \\
\hline \multirow{3}{*}{$\begin{array}{l}\text { Valoración del saber mapuche } \\
\text { en la escuela }\end{array}$} & Alumnos & 20 & 28 & 52 & 100 \\
\hline & Padres & 13 & 34 & 53 & 100 \\
\hline & Profesores & 14 & 62 & 24 & 100 \\
\hline \multirow{3}{*}{$\begin{array}{l}\text { La enseñanza escolar permite } \\
\text { conocer y apreciar a la } \\
\text { comunidad mapuche }\end{array}$} & Alumnos & 14 & 14 & 72 & 100 \\
\hline & Padres & 7 & 72 & 21 & 100 \\
\hline & Profesores & 4 & 29 & 67 & 100 \\
\hline \multirow{3}{*}{$\begin{array}{l}\text { Identificación mapuche en los } \\
\text { niños en el medio escolar }\end{array}$} & Alumnos & 9 & 18 & 73 & 100 \\
\hline & Padres & 10 & 14 & 76 & 100 \\
\hline & Profesores & 0 & 100 & 0 & 100 \\
\hline
\end{tabular}

Lengua y cultura mapuche se relacionan con el patrimonio cultural mapuche. El mapunzugun lengua mapuche constituye un medio de enseñanza y aprendizaje de contenidos educativos propios. Al preguntar si "Los profesores usan la lengua mapuche para enseñar las materias escolares", el 70\% de los alumnos, el $60 \%$ de los padres y el $53 \%$ de los profesores atribuyen una valoración en desacuerdo o muy en desacuerdo. Esto se interpreta como una ausencia real del mapunzugun en las prácticas pedagógicas de profesores que tienen por objeto la enseñanza de contenidos, sean mapuches o no mapuches, para la formación de alumnos que aún conservan un conocimiento sobre su cultura y lengua vernácula. En las escuelas que se consideran en la muestra, sobre el $90 \%$ del alumnado es de ascendencia mapuche, tomando como indicador sus apellidos y la mayoría de los miembros de las comunidades habitualmente socializan la tradición cultural haciendo uso del mapunzugun. Se constata que los profesores son conscientes sobre la ausencia del conocimiento mapuche asociado el uso de la lengua mapuche para enseñar las materias en clases.
Al interrogar sobre el domino de los profesores con respecto a conocimientos mapuches y el uso del mapunzugun en la enseñanza escolar, el $71 \%$ de los alumnos y el $51 \%$ de los padres atribuyen una valoración en desacuerdo o muy en desacuerdo. Esto significa que existe una escasa presencia del mapunzugun como conocimiento de dominio de los profesores y, por tanto, es muy difícil ser enseñado en la escuela. Sin embargo, sorprendentemente el $86 \%$ de los profesores atribuyen una valoración de acuerdo o muy de acuerdo con respecto al mismo aspecto. Esto significa que los alumnos pueden hablar el mapunzugun en clases, para comprender el conocimiento escolar. O bien, los profesores tendrían un dominio sobre conocimientos mapuches, pero que son ocultados o negados en la enseñanza escolar.

En la misma lógica del contexto sociocultural mapuche y el conocimiento que los niños poseen producto de la socialización en la familia y la comunidad, se pregunta sobre la idea de integrar el mapunzugun a la enseñanza escolar. Al respecto, el $73 \%$ de los alumnos, el $75 \%$ de los padres y el $70 \%$ de los profesores atribuyen una valoración de acuerdo o muy de 
acuerdo en que "Es importante que en la escuela se enseñe la lengua mapuche". Aquí se constata que en general los alumnos, padres y profesores aceptan y son conscientes sobre la necesidad de considerar contenidos y finalidades educativas mapuches para desarrollar procesos de escolarización en escuelas situadas en comunidades (Véase Tabla $\mathrm{N}^{\circ} 2$ ).

Tabla $N^{\circ}$ 2. El mapunzugun como herramienta para comunicar el conocimiento mapuche

\begin{tabular}{|c|c|c|c|c|c|}
\hline \multirow[b]{2}{*}{ Categorías } & \multirow[b]{2}{*}{ Actores } & \multicolumn{3}{|c|}{ Escala de valoración } & \multirow[b]{2}{*}{$\%$} \\
\hline & & No sé & $\begin{array}{c}\text { Muy en desacuerdo } \\
\text { o } \\
\text { En desacuerdo }\end{array}$ & $\begin{array}{c}\text { De acuerdo } \\
\text { o } \\
\text { Muy de acuerdo }\end{array}$ & \\
\hline \multirow{3}{*}{$\begin{array}{l}\text { Uso de la lengua mapuche en } \\
\text { la enseñanza escolar }\end{array}$} & Alumnos & 9 & 70 & 21 & 100 \\
\hline & Padres & 17 & 60 & 23 & 100 \\
\hline & Profesores & 23 & 53 & 24 & 100 \\
\hline \multirow{3}{*}{$\begin{array}{l}\text { Conocimiento y uso del } \\
\text { mapunzugun por parte de los } \\
\text { profesores }\end{array}$} & Alumnos & 14 & 71 & 15 & 100 \\
\hline & Padres & 11 & 51 & 38 & 100 \\
\hline & Profesores & 0 & 14 & 86 & 100 \\
\hline \multirow{3}{*}{$\begin{array}{l}\text { Importancia del uso del } \\
\text { mapunzugun en la escuela }\end{array}$} & Alumnos & 13 & 14 & 73 & 100 \\
\hline & Padres & 10 & 15 & 75 & 100 \\
\hline & Profesores & 6 & 24 & 70 & 100 \\
\hline
\end{tabular}

El contenido educativo mapuche se relaciona con saberes y conocimientos del patrimonio cultural que está en la base para la formación de persona y que es deseable de ser considerados como contenidos en la enseñanza escolar. Al interrogar si "Los profesores conocen saberes y conocimientos sobre el parentesco, la relación con la naturaleza y la religiosidad mapuche", el 55\% de los alumnos, el $58 \%$ de los padres y el $76 \%$ de los profesores atribuyen una valoración de acuerdo o muy de acuerdo. Esto significa que los profesores tienen conocimientos categorías de saberes mapuches, las cuales podrían ser integradas en las prácticas educativas escolares.

Con respecto a que "En la escuela se practica la cultura mapuche como el We Xipantu año nuevo mapuche, el palin deporte tradicional mapuche, similar al jockey sobre césped y el gürekan arte textil propiamente mapuche, el $75 \%$ de los alumnos, el $83 \%$ de los padres y el $90 \%$ de los profesores atribuyen una valoración de acuerdo o muy de acuerdo.
Esto se interpreta como una presencia real de aspectos del conocimiento cultural mapuche en la escuela y se observa su importancia para la formación de persona, ya sea en el medio familiar como en el medio escolar. Desde el punto de vista de los profesores significa que son conscientes de la importancia que tienen algunas prácticas de la tradición oral como el palin y el gübam, en la enseñanza escolar.

Al interrogar si "Los padres y familiares enseñan conocimientos mapuches, tales como la relación de parentesco y la relación con la naturaleza en mapunzugun", el $67 \%$ de los alumnos, el $64 \%$ de los padres y el $70 \%$ de los profesores atribuyen una valoración de acuerdo o muy de acuerdo. Esto se interpreta como una implicación directa de los padres y parientes en la transmisión del saber mapuche a niños y adolescentes. Al mismo tiempo que en la actualidad existen prácticas de transmisión del saber y conocimiento sociocultural en el medio familiar, haciendo uso del mapunzugun (Véase Tabla $\mathrm{N}^{\circ} 3$ ). 
Tabla $N^{\circ}$ 3. Presencia de saberes y conocimientos mapuches en el medio escolar

\begin{tabular}{|c|c|c|c|c|c|}
\hline \multirow[b]{2}{*}{ Categorías } & \multirow[b]{2}{*}{ Actores } & \multicolumn{3}{|c|}{ Escala de valoración } & \multirow[b]{2}{*}{$\%$} \\
\hline & & No sé & $\begin{array}{c}\text { En desacuerdo } \\
\text { o } \\
\text { Muy en desacuerdo }\end{array}$ & $\begin{array}{c}\text { De acuerdo } \\
\text { o } \\
\text { Muy de acuerdo }\end{array}$ & \\
\hline \multirow{3}{*}{$\begin{array}{l}\text { Dominio de saberes mapuche } \\
\text { por parte de profesores }\end{array}$} & Alumnos & 24 & 21 & 55 & 100 \\
\hline & Padres & 19 & 23 & 58 & 100 \\
\hline & Profesores & 5 & 19 & 76 & 100 \\
\hline \multirow{3}{*}{$\begin{array}{l}\text { Prácticas culturales mapuches } \\
\text { en la escuela }\end{array}$} & Alumnos & 8 & 17 & 75 & 100 \\
\hline & Padres & 8 & 9 & 83 & 100 \\
\hline & Profesores & 5 & 5 & 90 & 100 \\
\hline \multirow{3}{*}{$\begin{array}{l}\text { Enseñanza del conocimiento } \\
\text { cultural mapuche en el medio } \\
\text { escolar }\end{array}$} & Alumnos & 15 & 18 & 67 & 100 \\
\hline & Padres & 12 & 24 & 64 & 100 \\
\hline & Profesores & 7 & 23 & 70 & 100 \\
\hline
\end{tabular}

\section{DISCUSIÓN DE LOS RESULTADOS}

Desde el programa de Educación Intercultural Bilingüe (EIB) del Ministerio de Educación (MINEDUC), se define la educación intercultural en tres ámbitos de intervención vinculada con la acción docente: 1) Mejoramiento de las prácticas pedagógicas de profesores que se desempeñan en áreas con población indígena; 2) Incorporación de métodos de enseñanza y aprendizaje desarrollados por la familia y comunidades indígenas al proceso de formación de sus hijos; y 3) Generación de espacios de participación de los profesores con miembros de comunidades indígenas a fin de elaborar y construir materiales de enseñanza que integren saberes, técnicas y visiones de mundo al currículum y la gestión escolar (MINEDUC, 2005). Hoy día, la educación intercultural en Chile y en América Latina en general, en el marco de fuertes movimientos indígenas, ha hecho necesaria una reflexión en torno a las tensiones entre educación escolar y demandas indígenas, para la formación de persona en el medio escolar. Ello es cada vez más pertinente, en un contexto de globalización que hegemoniza y empuja al mundo para aceptar una racionalidad y un modelo de vida con un futuro enmarcado en el neoliberalis- mo. Este modelo de vida genera, a su vez, una creciente proliferación de identidades locales que no logran constituir un eje en relación con el marco cultural y social propio de los indígenas (Bonfil, 1993; Godenzzi, 1996b).

En ese marco, el concepto de interculturalidad hace referencia no solo del reconocimiento de la existencia de las culturas indígenas, sino que busca provocar un diálogo entre estas, una calidad de la enseñanza y aprendizaje en relación con el saber indígenaoccidental y a la inversa. Sin embargo, en el estudio se constata una polarización en la valoración del saber mapuche en la enseñanza escolar, asociada a la identificación de niños y jóvenes. Por una parte, los alumnos y profesores valoran de acuerdo o muy de acuerdo con respecto a la incorporación del conocimiento mapuche para la formación de persona en el medio escolar. Por otra parte, los padres atribuyen una valoración en desacuerdo o muy en desacuerdo con respecto a que la enseñanza escolar permite conocer y apreciar a la comunidad mapuche y que el conocimiento mapuche constituya un elemento de identificación en la escuela (Véase Tabla $N^{\circ} 1$ ). Los datos permiten asumir que en el medio escolar existen tensiones, conflictos y relaciones de poder 
susceptibles de ser modificados por la voluntad de los individuos. En efecto, es necesario asumir que el conocimiento cultural mapuche está presente en el medio escolar y que, por tanto, la relación dialógica a través de una educación intercultural que considere la lógica del conocimiento propio de los alumnos, se configuran como un proyecto de vida a construir y consolidar en forma permanente (Abdallah-Pretceille, 2001; Quintriqueo, 2007).

En ese contexto, la educación intercultural no es ni debe ser educación para los grupos diferenciados culturalmente, sino debe estar orientada a la comunidad educativa en su conjunto, para la formación escolar de sujetos de ascendencia mapuche y no mapuche, en una relación dialogante entre pueblos, los contenidos y finalidades educativas que la sustentan (Quilaqueo, Quintriqueo y Cárdenas, 2005). Es una educación que aspira a formar sujetos capaces de descubrir las diferencias, reconocerlas y comprenderlas dentro del carácter relacional de las culturas. Estas relaciones pueden ser y han sido muchas veces de conflicto. El aula intercultural, desde este paradigma, busca que los estudiantes puedan ver en las diferencias no solo una alternativa al modo de vida de la cultura de origen, sino también una alternativa posible de ser incorporada en la escuela y en la vida social. Esto significa, la valorización de la identidad, los contenidos y finalidades educativas de los otros, para la formación de persona; construcción de conocimientos y competencias dialógicas entre el saber social mapuche y occidental en el medio escolar.

La educación intercultural en el contexto regional (VIII, IX y X región) constituye una estrategia didáctica que tiene en cuenta paradigmas socioculturales mapuche y occidental en el marco de sus respectivas epistemologías que están en la base de la construcción de conocimientos educativos, desde ambas lógicas sociales y culturales en interrelación. Así, la educación intercultural se define como una herramienta y noción operativa (Küper, 1993; Godenzzi, 1996a), utilizada para designar las relaciones y encuentro de grupos humanos iguales, pero diversos (Nilo, 1999) en su manera de sentir, pensar y actuar. En este proceso se reconoce la identidad individual y social, diferenciada por elementos como la lengua, el territorio, una concepción de mundo y racionalidad con relación a la construcción de saberes y conocimientos propios. La dimensión bilingüe se entiende como una herramienta que permite comprender y estudiar saberes y conocimientos mapuches y no mapuches, para una mejor comunicación intercultural entre los miembros de sociedades con lógicas culturales diferentes. Sin embargo, en la perspectiva de la mayoría de alumnos, padres y profesores, en clases de escuelas consideradas en la muestra no se conoce o no se habla el mapunzugun, para comunicar contenidos y finalidades educativas mapuches que conservan una racionalidad distinta al conocimiento escolar. Aunque se constata en la valoración de todos los actores, una inclinación favorable sobre la importancia y necesidad de incorporar el uso y conocimiento del mapunzugun asociado a la enseñanza de conocimientos culturales mapuches, desde una educación intercultural y bilingüe (Véase Tabla $\mathrm{N}^{\circ} 2$ ).

Entonces, uno de los principales obstáculos para el desarrollo de la educación intercultural bilingüe, se relaciona con la internalización de ideologías y creencias profundamente arraigadas, tanto de las sociedades dominantes como de los Pueblos Indígenas (Moya, 1996). Existe un ideal de monolingüismo como componente de un Estado nacional homogéneo y un prejuicio sobre la supuesta inferioridad de las lenguas y culturas indígenas y su consecuente incapacidad de transformarse en instrumentos eficientes de procesos educativos modernos y de alta calidad. Prevalece una concepción de bilingüismo sustractivo, fundamentado en la creencia que para aprender bien la lengua nacional hay que abandonar y olvidar la lengua indígena propia; y que si los niños ya hablan más o menos bien el castellano, no conviene 'regresar al pasado' y permitir que aprendan y pierdan el tiempo con las lenguas ancestrales.

En la actualidad, en el desarrollo del currículum escolar existe un desafío para atenuar conflictos sociocognitivos e identitarios en contextos donde coexisten la racionalidad mapuche y no mapuche, en la construcción del saber y conocimiento educativo en el nivel de Enseñanza Básica ¿Es posible superar el problema de conflicto sociocognitivo e identitario de alumnos de ascendencia mapuche? 
¿En contexto mapuche existe la apertura del profesorado y de quienes tienen a cargo la gestión educativa, para incorporar las expectativas locales sobre la formación de persona desde un enfoque educativo intercultural que tiene por objeto de colaboración activo-participativa de la familia y la comunidad mapuche en el desarrollo de actividades educativas escolares? En el estudio se constata que en general los alumnos, padres y profesores valoran positivamente el conocimiento mapuche que poseen los profesores, las prácticas culturales mapuches que se desarrollan en el medio escolar, lo cual favorecería una educación intercultural (Véase Tabla $N^{\circ} 3$ ).

La escuela es considerada como espacio educativo por excelencia, la sala de clases, con diversas variantes de organización en función de actividades que se desarrollen ¿la escuela como institución del Estado, responsable de la formación de niños y adolescentes en las dimensiones física, social y cultural, muestra apertura a la participación comunitaria en los procesos educativos? De acuerdo a los datos, la comunicación entre escuela y comunidad ha sido solo circunstancial, accidental y superficial, lo cual persiste hasta hoy día. Esta situación es contradictoria con las propuestas del Ministerio de Educación que postula a una escuela abierta a la relación con la comunidad, donde se amplían los horizontes de la escuela, produciéndose relaciones comunicativas reversibles, esto es, EscuelaComunidad - Comunidad-Escuela, generando una nueva concepción de espacio educativo.

La formación escolar de niños y adolescentes se inscribe en un contexto donde coexisten racionalidades del saber mapuche y no mapuche. Esto supone un profesor que comprende estas racionalidades y que conoce los saberes educativos de ambas racionalidades, para orientar una educación fundada en conocimientos propios (Quintriqueo, 2008). Este supuesto pone en evidencia la necesidad de establecer una relación entre el saber docente (Gauthier et al., 1997) y el saber mapuche, en procesos educativos de escuelas que se sitúan en contexto de comunidades mapuches. En este sentido, habría que preguntarse ien qué incide una relación entre el saber educativo occidental y mapuche en el aprendizaje escolar? Además, si en la formación escolar, en las actividades de enseñanza se explicitan las relaciones de poder entre la cultura mapuche y no mapuche, con el fin de plantear una relación intercultural desde el saber y conocimiento propio de las comunidades. La educación intercultural debería ser capaz de conjugar saberes, puesto que no se trata de una educación para indígenas, por indígenas, acerca de conocimientos indígenas. Entonces, debe propender a la vinculación de la ciencia universal con los saberes y conocimientos cotidianos, en el marco de una educación que propicie la construcción de conceptos, procedimientos y actitudes científicas, estableciendo una relación con los diferentes sistemas de entendimiento sobre la realidad.

\section{CONCLUSIONES}

Los contenidos de un currículum intercultural radican en la forma de concebir la formación, en relación con orientaciones precisas para comprender las lógicas de pensamientos, saberes y conocimientos educativos que están en la base. La transmisión de estos contenidos permite a las nuevas generaciones desenvolverse en el marco de la sociedad que ha establecido un conjunto de disposiciones que la persona debe desarrollar en su identidad individual y social, en relación con los conocimientos del patrimonio cultural mapuche y no mapuche. En efecto, el currículum intercultural tiene como finalidad la organización y sistematización de contenidos educativos propios de los educandos, transmitidos a partir de la socialización en el medio familiar y comunitario.

En los Pueblos Indígenas, en general, el saber y conocimiento están en relación directa con el mundo natural, la relación de parentesco en la comunidad y el conocimiento sobre el universo. Estos saberes son construidos a través del desarrollo histórico de los pueblos. Es el producto de un proceso de creación mental y cognitiva que configura el patrimonio cultural objetivo y subjetivo. De esta manera, el patrimonio cultural mapuche se constituye por elementos como: valores, creencias, normas, reglas, saberes y conocimientos propios asociados al patrimonio cultural objetivo (tecnológico) y subjetivo (pensamiento), que por medio de una lógica contribuyen al desarrollo de la familia y la comunidad. Estos saberes se asumen como el 
fundamento de los contenidos educativos para la enseñanza y el aprendizaje intercultural de niños mapuches y no mapuches.

Entonces, el profesor no puede contar únicamente con los saberes formalizados para el desarrollo de su clase. Por una parte, la práctica pedagógica es mucho más compleja que eso, está muy implicada por la contingencia y su relación con los contenidos que derivan de las ciencias. Por otra, su primer propósito no es el conocimiento, sino la acción. Es decir, la práctica de la enseñanza no se relaciona necesariamente con un conocimiento teórico, sino más bien con una deliberación práctica. Este razonamiento práctico que se apoya sobre una base poco definida, discutible con relación al orden de los valores, obliga necesariamente al profesor a hacer un espacio a la prudencia sobre la cual orienta su acción. En esta lógica de razonamiento, la situación pedagógica es multidimensional e impredecible, ligada a una realidad compleja que lo compone. La enseñanza del profesor se relaciona con un devenir y una acción fundada en la incertidumbre, donde todo requiere y necesita a la vez de recursos de inteligencia científica y los recursos del saber popular e indígena.

Aunque históricamente en contexto indígena, pero principalmente en comunidades mapuches, la educación escolar presupone un determinado concepto de niñez, una determinada racionalidad del conocimiento y lógica del pensamiento para el aprendizaje fundado en el marco social y cultural occidental. Sin embargo, los niños tienen una formación en base a conocimientos educativos propios que le permiten la construcción de la identidad sociocultural mapuche, aspecto desconocido en las escuelas y en la sociedad dominante al momento de definir contenidos y finalidades educativas, sea en forma consciente o inconsciente. En este proceso, la naturaleza, descontextualizada del aprendizaje escolar, oculta el valor pedagógico del saber y conocimiento educativo propio de los alumnos. Entonces, se constata una distancia epistemológica entre la racionalidad mapuche y occidental, tanto en la construcción del conocimiento como el pensamiento para comprender el mundo. Estas racionalidades están en la base del proceso de formación de persona, sea en el ámbito familiar o escolar. La escuela está en el centro de la distancia epistemológica, donde la formación de persona es el nexo de una relación educativa hipotética de ambas racionalidades, históricamente en oposición. Superar esta distancia es el objetivo de la educación y currículum intercultural, para generar una relación dialógica entre el saber mapuche y occidental en la formación de persona.

Desde un marco general, una propuesta de educación intercultural debiera asumir los siguientes supuestos: 1) En relación con el proceso de enseñanza-aprendizaje se asume que las personas adquieren habilidades y destrezas que le permiten vivir en una determinada comunidad, en la cual están inmersos; 2 ) Las destrezas y habilidades se relacionan con sistemas de significados que capacitan a las personas para comunicarse y con actividades prácticas que se orientan hacia la transformación del mundo natural, social y cultural; 3) Quienes participan de dichas situaciones lo hacen desde sus propias perspectivas y metas que son iguales, pero diferentes en el sentido y significado atribuido a las cosas; 4) Las personas aprenden inmersas en un mundo social, aunque el acto de aprender se considere como un hecho predominantemente individual; 5) Las personas aprenden en la diferencia, lo cual constituye una riqueza de la producción humana, para mejorar la calidad de los aprendizajes, las oportunidades y la convivencia social; y 6) Las prácticas pedagógicas interculturales tienen por objeto la generación de relación dialógica entre saberes populares, indígenas y el saber y conocimiento escolar. 


\section{BIBLIOGRAFÍA}

ABDALLAH-PRETCEILLE, M. (2001). La educación intercultural. Barcelona: IDEA BOOKS, S.A.

BISQUERRA, R. (2004). Metodología de la investigación educativa. Madrid: LA MURALLA, S.A.

BONAL, X. (1998). Sociología de la educación: Una aproximación crítica a las corrientes contemporáneas. Barcelona: Paidós.

BONFIL, G. (1993). La teoría del control cultural en el estudio de los procesos étnicos. Revista Papeles de la Casa Chata. Año 2, N ${ }^{\circ}$, México, pp. 34-65.

BRUNNER, J. J. (1998). Malestar en la sociedad chilena: ¿De qué, exactamente, estamos hablando? Santiago de Chile: Centro de Estudios Públicos.

CANALES P. (1998). Escuelas chilenas en contexto mapuche. Integración y resistencia, 1860-1950. En Revista Última Década, Centro de Investigaciones Pedagógicas de Viña del Mar, $\mathrm{N}^{\circ}$ 9, pp. 9-23.

CATRIQUIR, D. Y DURÁN, T. (1997). Reformas Educativas en Chile desde la perspectiva interétnica. De la sorpresa a la reflexión mapuche. En Pueblos indígenas y Educación. $\mathrm{N}^{\circ}$ 37-38. pp. 111-141.

CAÑULEF E. (1998). Introducción a la Educación Intercultural Bilingüe en Chile. Temuco: Editorial Pillán.

CHIODI, F. (1990). La educación indígena en América Latina. México, Guatemala, Ecuador, Perú y Bolivia. Quito. Abya-yala-Unesco. Tomos I y II.

CHIODI, F. Y BAHAMONDES, M. (2002). Una escuela: diferentes culturas. Santiago de Chile: LOM Ediciones.

EGAÑA, M. (2000). La educación primaria popular en el siglo XIX en Chile: Una práctica de política estatal. Santiago de Chile: LOM Ediciones.

FERNÁNDEZ, F. (2003). Sociología de la Educación. Madrid: Editorial PEARSON EDUCACIÓN S.A.
FOERSTER, R. (1988). Organizaciones, líderes y contiendas mapuche (1900-1970). Santiago de Chile: Ediciones Mundo.

FORNET-BETANCOURT R. (2001). Filosofía e interculturalidad en América Latina, Intento de introducción no filosófica. En Heise M. (ed.) INTERCULTURALIDAD; Creacción de un concepto y desarrollo de una actitud. Lima: Inversiones Hathuey S.A.C., pp. 63-73.

FORQUIN, J. C. (1997). Les sociologues de l'éducation américains et britanniques. Présentation et choix de textes. Bruxelles: De Boeck Université.

GAUTHIER, B. (1998). Recherche sociale. De la problématique à la collecte des données. TseFoy: Presses de l'Université du Québec.

GAUTHIER, C.; DESBIENS, J.; MALO, A.; MARTINEAU, S. ET SIMARD, D. (1997). Pour un Théorie de la Pédagogie. Recherches Contemporaines sur le savoir de enseingants. Québec: Le Presses de l'Université Laval.

GODENZZI, J. (1996a). Construyendo la convivencia y el entendimiento: educación intercultural en América Latina. Cuzco: Centro de Estudios Regionales Andinos Bartolomé de Las Casas.

GODENZZI, J. (1996b). Educación e interculturalidad en los Andes y la Amazonía. Cuzco: Centro de Estudios Regionales Andinos Bartolomé de Las Casas.

KÜPER, W. (1993). Investigación pedagógica intercultural bilingüe. Quito: ABYA-YALA.

KRIPPENDORFF, K. (1990). Metodología de análisis de contenido. Teoría y práctica. Barcelona: Paidós.

LABARCA, A. (1939): Historia de la Enseñanza en Chile. Santiago de Chile Editorial Universitaria.

MAGENDZO, A. (1986). Currículum y Cultura en América Latina. Santiago de Chile : Academia de Humanismo Cristiano. PIIE.

MAGENDZO, A. (2007). Homogeneidad y heterogeneidad curricular: Un tema vinculado con la centralización y la descentralización del currículum. Documento de trabajo. 16 de enero de 2007.

MOYA, R. (1996). Conceptos políticos y estra- 
tegias para la educación bilingüe en América Latina. En Catriquir D. (ed.) Actas 1er Seminario Latinoamericano de Educación Intercultural Bilingüe, Universidad Católica de Temuco, pp. 43-68.

NILO, S. (1999). Los desafíos de la interculturalidad: oportunidades y tareas para la educación formal. En Quilaqueo, D. (ed.) Educación Intercultural Bilingüe. Actas Segundo Seminario Latinoamericano. Temuco, Universidad Católica de Temuco, Vicerrectoría Académica, pp. 147-156.

PERRENOUD, PH. (2005). L'école face à la diversité des cultures. La pédagogie différenciée entre exigence d'égalité et droit à la différence. Faculté de Psychologie et des Sciences de l'Éducation Université de Genève.

QUILAQUEO, D.; QUINTRIQUEO, S. Y CÁRDENAS, P. (2005). Educación, currículum e interculturalidad. Elementos sobre formación de profesores en contexto mapuche. Santiago de Chile: Frasis Editores.

QUILAQUEO D. Y QUINTRIQUEO S. (2008). Formación docente en educación intercultural para contexto mapuche en Chile. Revista Cuadernos Interculturales. Primer semestre 2008, Año 6, número 10, pp. 91-110.

QUINTRIQUEO S. Y MAHEUX G. (2004). Exploración del conocimiento sobre la relación de parentesco como contenido educativo para un currículum escolar intercultural en comunidades mapuche. Revista de Psicología, Universidad de Chile, Vol. XIII, N 1, 73-91.

QUINTRIQUEO, S. (2007): "Implicancias de un modelo curricular monocultural en la construcción de la identidad sociocultural de alumnos/ as mapuches de la IX Región de La Araucanía". Tesis para optar al grado de Doctor en Educación. España: Universidad de Extremadura.

QUINTRIQUEO, S. y MCGINITY MARGARET (2008). "Currículum monocultural en la construcción de la identidad sociocultural de alumnos/as de ascendencia mapuche". ORGANIZACIÓN Y GESTIÓN EDUCATIVA. Número LXXII, Año XVI, Vol. $N^{\circ} 4$, julio y agosto de 2008, pp. 34-35.
QUIVY R. Y CAMPENHOUDT, L. (1998). Manual de Investigación en Ciencias Sociales. México: Edición Limusa.

ROCKWELL, E. (1995). La escuela cotidiana. México: Fondo de Cultura Económica.

TEDESCO, J. (2000). Educar en la sociedad del conocimiento. Ciudad de México: Fondo de Cultura Económica.

UNESCO (2005). La discriminación y el pluralismo cultural en la Escuela. Casos de Brasil, Chile, Colombia, México y Perú. Organización de las Naciones Unidas para la Educación, la Ciencia y la Cultura. Santiago de Chile: Oficina Regional de Educación para América Latina y el Caribe, OREALC/UNESCO.

\section{DOCUMENTOS DE TRABAJO}

INSTITUTO INDIGENISTA INTERAMERICANO (1996). Convenio $N^{\circ} 169$ sobre Pueblos Indígenas y Tribales en Países Independientes, 1989. Organización Internacional del Trabajo OIT. En América Indígena, Volumen LVIII, $\mathrm{N}^{\circ}$ 3-4.

INSTITUTO INDIGENISTA INTERAMERICANO (1996). Proyecto de Declaración Universal de los Derechos de los Pueblos Indígenas. Organización de Naciones Unidas, ONU, abril de 1994. En Revista América Indígena, Volumen LVIII, N³-4, julio y diciembre de 1996.

LEY INDÍGENA (1993). Ley $N^{\circ}$ 19. 253 D.Of. $5^{\circ}$ $10^{\circ}$ 1993. Temuco: CONADI.

MINISTERIO DE EDUCACIÓN DE CHILE (MINEDUC) (1990). Ley Orgánica Constitucional de Enseñanza. Santiago de Chile.

MINISTERIO DE EDUCACIÓN DE CHILE (MINEDUC) (2004/2005). Orientaciones para la contextualización de Planes y Programas para la Educación Intercultural Bilingüe. Santiago de Chile.

MINISTERIO DE PLANIFICACIÓN DE CHILE (MIDEPLAN) (2004). Verdad Histórica en la relación mapuche y no mapuche. Santiago de Chile. 